\title{
Study on the Countermeasures for Disposing of the Water-rich Section of Junmachang Tunnel
}

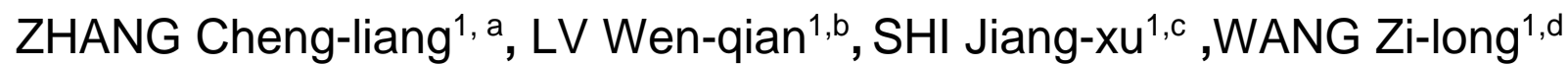 \\ ${ }^{1}$ Faculty of Land Resources Engineering, Kunming University of Science and Technology, Kunming, \\ Yunnan, China 650093

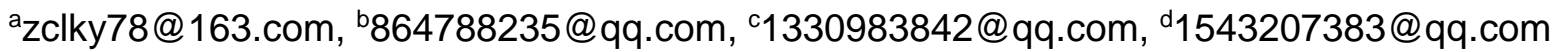

\begin{abstract}
Keywords: Enriched water in surrounding rocks, Softening effect, Grouting, Reinforcement, Waterproof and drainage, Stability

Abstract. During tunnel construction, the stability of tunnel surrounding rock caused by excavation of water-rich section has always been a major problem in the project. In this paper, the influx of water in the face of the excavation of the water-rich section of the Junmachang tunnel caused a large amount of water to be unable to be constructed. The on-site sampling was carried out to test the natural and saturated water conditions and the rock mass of the excavation section under the water-rich effect was determined. The characteristics of softening of body mechanics, in view of the lithology of the surrounding rocks of the tunnel and the water inrush of groundwater in the tunnels in the water-rich section of the tunnel, a combination of the grouting reinforcement of the advancement of the small face of the tunnel face, the radial grouting reinforcement around the tunnel, and the combination of a reasonable anti-drainage system were proposed to close the groundwater treatment. The results show that the deformation of the surrounding rock in the water-rich section of the tunnel after treatment is controlled within a reasonable deformation range, and the accumulative total settlement of the dome settlement and the cumulative convergence of the clearance are less than $1 / 3$ of the reserved deformation. The water-rich section of the Junmachang has achieved good results in the combination of advanced small-vessel grouting reinforcement, peripheral radial grouting reinforcement, and the installation of a reasonable anti-drainage system, which has certain engineering application value.
\end{abstract}

\section{Introduction}

During the construction of mountain tunnels, the tunnel construction caused by the effect of groundwater on the surrounding rock often faces difficulties[1-5]. When the surrounding rocks are water-rich, the excavation of tunnels in the water-rich section will lead to large deformation of the surrounding rock, settlement joints on the surface, damaged supporting structures, and even collapse, affecting the safety construction and restricting the construction of the tunnel construction schedule. For the tunnel excavation construction of surrounding rocks in the water-rich section, reasonable reinforcement measures are taken to limit the weakening of the surrounding rock by groundwater, and pre-reinforcement and pre-treatment measures are taken before the occurrence of the disease, which is more practical for the tunnel construction in the water-rich section.

A number of studies have been carried out on the characteristics, construction, and reinforcement of the surrounding rock of the water-rich section of the tunnel. Wei Xing[6] and others responded to flooding, collapse, surface cracking, and groundwater level drop in the Beigang soft rock tunnel in the flooded section. Through indoor model tests, the damages were reconstructed and the influencing factors and laws of the disease were studied. Guo Xiaohong[7] analyzed the causes of serious deformation of the initial support of the soft and rich water-rich zone of the Dakui tunnel from the aspects of geological conditions, gushing water characteristics, and construction methods, and studied the support face and failure section scheme. Wang Ying[8] and others combined with fracture mechanics found that the I-II composite cracks produced by the rock under hydraulic fracturing were further connected under high water pressure, blasting and other actions and eventually led to disasters. Deng Renqing[9] made detailed introduction and practical application of curtain grouting and water shut-off technology in the rich water area of Leshan Tunnel. 
At present, most of the studies on the surrounding rocks of the tunnels in the water-rich section of tunnels are reinforced and disposed after the tunnels are damaged. However, there are relatively few researches on the pretreatment and pre-reinforcement of the surrounding rocks before the disaster. In the surrounding area of the water-rich section, under the long-term hydraulic action of the groundwater, the strength of the surrounding rock is significantly reduced. Under the long-term effect of the water pressure, some micro-fissures in the rock gradually penetrate and further increase the weakening effect of the groundwater on the surrounding rock of the tunnel. It is more meaningful to adopt reasonable pre-reinforcement measures for the surrounding rocks of the water-rich section before the disease to ensure safe and orderly tunnel excavation.

\section{Project Overview}

The Junmachang Tunnel is a control project of Shanghe Expressway. It uses two-way four-lane construction. The cumulative total length of the tunnel is $519,9.92 \mathrm{~m}$ and the design speed is $80 \mathrm{~km} / \mathrm{h}$. In the tunnel area, the terrain slope changes greatly, and the relative height difference of the surface is $178.75 \mathrm{~m}$. The tunnel body is dominated by gravelly silty clay, gravel, and strong medium weathered basalt. The geological conditions of the tunnel's rich water section are relatively complex and the rock is relatively broken. The underground water in the tunnel area is distributed with the quaternary pore water and the bedrock fissure water. In the water-rich section of the tunnel, the surrounding rock is of $\mathrm{V}$ grade. The integrity and stability of the surrounding rock are poor, the joints and fissures are developed, the rock body is fractured, and deformation or even collapse occurs after excavation. When the tunnel was excavated to the position of $\mathrm{K} 4+850$, there was a large gush of water in the face, and the amount of water in the excavated face and the excavated section was greater than $300 \mathrm{~L} / \mathrm{min}$, and continued for 1 week, making it difficult to excavate the face. Therefore, high-density electric probing was carried out in the K4+250 K4+850 section of the Junmachang tunnel exit, and the unfavorable geological range, properties and formation causes of the tunnel K4+250 K4+850 sections were identified in detail, and studied in the water-rich section. The characteristics of the mechanical properties of the surrounding rock provide the basis for tunnel excavation.

\section{The Weakening Effect of Water on Rocks}

The intrusion of groundwater will change the physical and mechanical properties of the rock, and its weakening will lead to a decrease in the strength of the rock, thus resulting in a decrease in the self-supporting capacity of the surrounding rock. The rock samples were taken from the tunnel's rich water section and made into test specimens for testing. Comparing the uniaxial compressive strength of the sample under natural and saturated conditions, and comparing the rock physical and mechanical parameters of the wet and dry specimen elastic modulus measured by the uniaxial compression deformation test of unconstrained rock, determine the weakening effect of rock mass caused by the water. The compressive test results of the rock mass under natural and saturated conditions are shown in Fig. 1, and the elastic modulus test results are shown in Fig. 2.

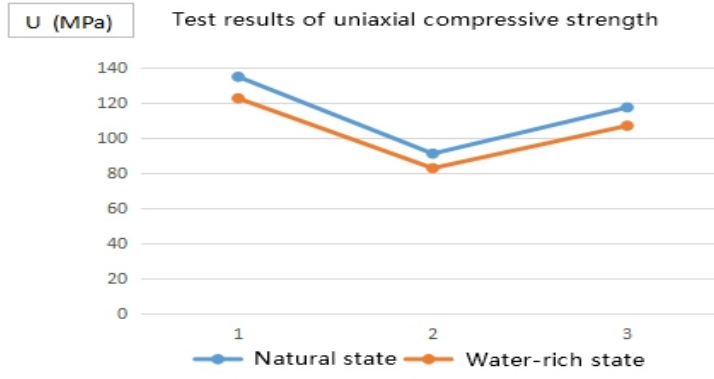

Fig. 1 Uniaxial compressive strength test results under different conditions 


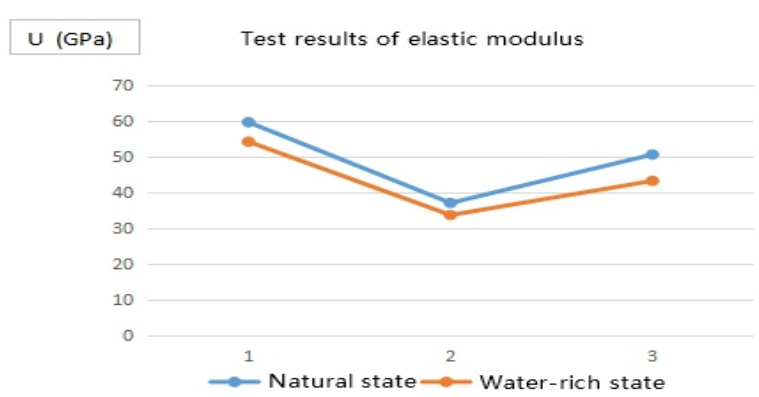

Fig. 2 Rock elastic modulus test results under different conditions

The uniaxial compressive strength and modulus of elasticity of the rock mass were measured under natural and saturated conditions in the laboratory. The water from dry to saturated state had a significant effect on the weakening of the rock specimen strength. The softening coefficient of the rock specimen is about 0.86 . The result shows that the strength of the rock body decreases rapidly under the effect of water enrichment and it is more likely to lose stability after excavation. Because the existence of a large number of joint cracks in the rock mass results in a smaller actual softening coefficient, the softening effect becomes more pronounced.

\section{Tunnel Water-rich Section Treatment Countermeasures}

Due to the weakening effect of groundwater on the surrounding rock, the self-stability of the surrounding rock is even worse. Therefore, in order to ensure the safety of construction, appropriate support measures should be taken during the excavation process in accordance with the differences in the type of surrounding rock and the occurrence of groundwater. Through engineering experience analogy, the excavation of the tunnel was carried out using the three-step and seven-step method of core soil excavation. During the excavation operation of the water-rich section, the advanced small pipe and radial grouting were used to carry the surrounding rock of the rich water segment.

Advance Small Catheter Grouting. The underground water of the Junmachang tunnel water-rich section is rich in groundwater, especially in the front of the face of the palm. It forms a large strand of water after excavation. Therefore, the surrounding rock in front of the face must be reinforced before excavation to prevent palm from excavation. Collapse near the subsurface or cause the tunnel to be overturned. In the construction of the tunnel's water-rich section, the hot-rolled seamless steel pipe of $\Phi 42 \times 4$ is used for the small grouting pipe for advance grouting. The length of the grouting small pipe is $4.5 \mathrm{~m}$. The front of the steel pipe needs to be processed into a tapered shape to facilitate the penetration into surrounding rock. A $\Phi 8 \mathrm{~mm}$ stiffener hoop was welded to the tail of the small slurry pipe. A $15 \mathrm{~mm}$ diameter squeezing hole with a plum shape was drilled every $15 \mathrm{~cm}$ around the pipe wall. The grouting ducts are arranged in a plum pattern, and the ducts are laid in the tunnel dome about 120 degrees. Before the grouting of the small pipe is advanced, the use of moulded concrete within $5 \mathrm{~m}$ of the tunnel excavation surface and both sides of the tunnel shall be used to close or inject 50 100mm thick reinforced concrete surrounding rock.

The water-rich section of the Junmachang tunnel is grouted with a cement-water glass double slurry. The spread radius of the slurry during grouting is not less than $0.5 \mathrm{~m}$, the water-cement ratio of the grouting cement slurry is $1: 1$, and the grouting pressure is $0.5-1.0 \mathrm{Mpa}$ between. During grouting, the slurry is injected from the orifice at one time. In order to ensure that the slurry in the small conduit is full and compact, it is necessary to wait until the slurry exits from the exhaust hole and finally grouting, when the grouting pressure is reached or the injection pressure is met. The grouting was terminated when the slurry amount. In order to allow the slurry to have sufficient time to complete the gelling and achieve the goal of reinforcing the surrounding rock in front of the face, the excavation of the tunnel shall be conducted 4 hours after the completion of the grouting.

Radial Grouting Reinforcement Around the Tunnel. After the tunnel is excavated, the tunnel is affected by the excavation disturbance within a certain range, the stress is weak, and the surrounding groundwater is more likely to cause collapse. It is necessary to fully grouting the surrounding tunnel 
after the excavation. Slurries can be used to increase the load-bearing capacity of the surrounding rock and improve the stress conditions of the tunnel support structure. Due to the poor stability of the surrounding rocks of the tunnels in the water-rich section of the tunnel, there are generally raindrop-shaped drips. Therefore, a full-section radial grouting with a reinforced ring of $4 \mathrm{~m}$ is adopted in the water-rich section to control the deformation of the surrounding rock and ensure the stability of the surrounding rock.

Peripheral radial reinforcement grouting is carried out using $\Phi 25$ system bolts. A single-fluid grouting experiment before grouting can simplify the grouting process, reduce the cost, and increase the consolidation strength. The surrounding radial full-surface reinforcement grouting liquid is mainly made of cement, and $5 \%$ by weight of water glass is added to achieve the purpose of better consolidation of surrounding rocks. The end of grouting is based on the design of a single pipe to meet the design grouting quantity. In the grouting project, attention should be paid to the observation of the working face of the tunnel and the state of initial support to ensure safety.

Tunnel Waterproof and Drainage System. The advancement of small-concrete grouting reinforcement and peripheral radial grouting reinforcement is through grouting to form a bearing body consisting of slurry and surrounding rock. It enhances the self-stabilizing ability by increasing the strength of the surrounding rock and weakening the permeability of groundwater around the tunnel. The unified drainage and discharge of groundwater near the tunnel was discharged through the drainage facilities to reduce the continuous impact of groundwater on the stability of the tunnel.The water-rich section of the Junmachang Tunnel adopts a combination of "prevention, drainage, plugging, and interception" to treat groundwater and form a perfect internal and external drainage system. The drainage system is composed of a circular drainage blind pipe, a horizontal water guide pipe, a longitudinal wall drain pipe, and a road drain. The ring drainage blind pipe is set at about $3 \mathrm{~m}$ around the water-rich section of the surrounding rock, which can increase when the local water volume is large; when the tunnel continues to excavate and continues to seep water, a $\Phi 75 \mathrm{~mm}$ single-wall drilling hole can be laid along the surface of the surrounding rock. One to three bellows guide the drainage, and at the same time to ensure that the drainage pipe can be in contact with the rock face, you can spray $2 \sim 5 \mathrm{~cm}$ thick concrete on the surrounding rock surface. Vertical drainage holes are provided for the vertical drainage blind pipes at both sides of the wall at both sides of the wall. The horizontal aqueducts are installed along the bottom of the tunnel at a distance of $10 \mathrm{~m}$. They connect the longitudinal drain pipes to the drainage ditch and use $\Phi 110 \mathrm{~mm}$ PE double-walled corrugated pipes.

\section{Project verification}

On the K4+600 K4+850 section of the right tunnel of the water-rich section, the stability monitoring of the excavation surrounding rock was conducted with advanced grouting, circular grouting and combined drainage of the system. From the monitoring results, the accumulated amount of subsidence of each section before the tunnel liner lining is less than one-third of the maximum reserved deformation, that is $5 \mathrm{~cm}$, and the displacement rate is less than $4.20 \mathrm{~mm} / \mathrm{d}$; the cross-section of each section before the tunnel is watered that the convergent cumulative values tend to be stable and the cumulative convergence value is less than one-third of the maximum reserved deformation, that is $5 \mathrm{~cm}$, and the displacement rate is less than $2.42 \mathrm{~mm} / \mathrm{d}$. The maximum curve of the crown sinking in the section of the right section of the tunnel K4+600 K4+850 is shown in Figure. 6. The tunnel YK4+600 YK4+850 is rich in water. After the tunnel excavation disturbs the surrounding rock, pore water and bedrock fissure water in the surface of the rock easily migrate along the crack passage toward the face of the face, making the surface around the face of the mantle. Fissure water produces convergence, which greatly increases water content. From the above figure, we can see that in the excavation of the segment to take the lead face to take the small catheter, radial reinforcement after the excavation, optimize the drainage system measures enough, the deformation of the surrounding rock has been effectively controlled, under the tunnel dome and the cumulative 
maximum value of complies with relevant standards, and the surrounding rock of the tunnel has basically stabilized and achieved certain results.

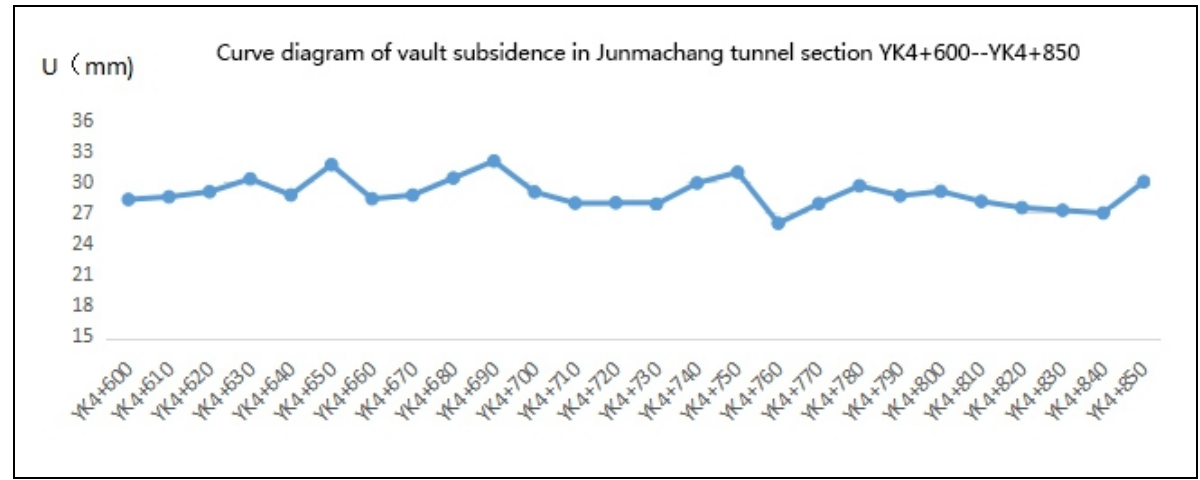

Fig. 3 Maximum curve diagram of vault subsidence in Junmachang tunnel section YK4+600 YK4+850

\section{Conclusion}

The strength of the surrounding rock in the water-rich section of the Junmachang Tunnel dropped rapidly, and the amount of water in the face was large, making it difficult to continue construction. Sampling of rock samples from the site was performed to test the mechanical properties of the rock under natural and saturated conditions. Combined with the geological conditions of the water-rich section of the tunnel, the grouting reinforcement of the small-lead pipe ahead of the tunnel face, the radial grouting reinforcement around the tunnel, and layout were adopted. The combination of a reasonable anti-drainage system and the reinforcement of the water-rich section led to the following conclusions.

(1) Sampling and making rock mechanical properties test from the site, the strength of the sample under natural and saturated conditions is significantly reduced, and the softening coefficient is about 0.86. Combined with the existence of a large number of joint fractures and even cracks in the surrounding rock of the site, the effect of the surrounding rock on the softening of groundwater is even more obvious.

(2) The surrounding rocks of the tunnels in the water-rich section of the tunnel, under the influence of the hydraulic effect of the groundwater and its permeability, the strength of the surrounding rock is significantly reduced, and it is easy to cause collapse after excavation, and appropriate pre-reinforcement and pretreatment measures are taken to deform the surrounding rock. Control is more effective within a reasonable range.

(3) For the water-rich section, a combination of advanced small-conduit reinforcement, peripheral radial grouting reinforcement, and a reasonable anti-drainage system was adopted. The groundwater during the entire excavation process was strictly controlled and achieved good results. After the reinforcement, the surrounding rock dome and radial cumulative deformation are all less than 1/3 of the reserved deformation. The practice has proved that the disposal of the water-rich section is effective.

\section{Acknowledgements}

This work was financially supported by the National Natural Science Foundation of China (51264018).

\section{References}

[1] Li S, Xie Y, Zhu X. Research on countermeasure of water gushing with collapse in process of Wushaoling highway tunnel crossing F4 fault fracture zone[J]. Chinese Journal of Rock Mechanics \& Engineering, 2013, 32:3602-3609. 
[2] Zuo Qingjun, Wu Li, Lin Cunyou et al. Analysis of collapse mechanism and its treatment measures in the fault zone of Fushui soft rock tunnel[J]. Chinese Journal of Rock Mechanics and Engineering, 2016, 35(2):21-21.

[3] Tan Zhongsheng,Wang Xiuying,Wan Fei et al. Research on prevention and control technology system of sudden water inflow of Guanjiao tunnel[J]. China Civil Engineering Journal, 2017(s2):1-7.

[4] Zheng Xiaofu. Analysis of tunnel gushing water treatment and compulsory drainage method [J]. Western Exploration Engineering, 2018 (5):188-191.

[5] Li Wenyang. Discussion on the Causes of Floor Water Gushing in Huayingshan Tunnel [J].Technology of Highway and Transport,2016,32(6):106-109.

[6] Wei Xing, Shen Le, Tao Zhiping. Model test studies of collapse and settlement of tunnel in saturated soft rocks [J]. Rock and Soil Mechanics, 2012, 33(8):2291-2296.

[7] GUO Xiaohong, CHEN Feifei, GENG Yiyi et al. Research on support techniques for tunnel in watery and weak stratum [J]. Rock and Soil Mechanics, 2011(s2):449-454.

[8] Wang Ying, Chen Qiang, Wei Youyi et al. Water/Rock Interaction Mechanism in Deep-buried Tunnels in Karst Area [J]. China Railway Science, 2004, 25(4):55-58.

[9] Deng Renqing. Construction A Method of Water Blocking by Grouting in High Pressure and Enriched Water Tunnel and Its Application [J]. Chinese Journal of Underground Space and Engineering, 2006, 2(2): 263-266. 